\section{International Scientific Journal Theoretical \& Applied Science}

p-ISSN: 2308-4944 (print) e-ISSN: 2409-0085 (online)

Year: $2016 \quad$ Issue: 12 Volume: 44

Published: $15.12 .2016 \quad$ http://T-Science.org

SECTION 20. Medicine.
Said Abdullaevich Salekhov

Professor, doctor of medical sciences

Novgorod State University of Yaroslav Mudry, Russia ssalehov@mail.ru

Bakytgul Rakhimbekovna Nurmukhambetova $\mathrm{PhD}$, assistant of the Department Kazakh National Medical University named after SD Asfendiyarov bahytgul1961@mail.ru

Marina Vladislavovna Konovalova $\mathrm{PhD}$, Associate Professor, Department of Obstetrics and Gynecology, St. Reterburgsky State Pediatric

Medical University marinavladi73@mail.ru

Tatyana Ivanovna Prohorovich $\mathrm{PhD}$, Associate Professor, Department of Obstetrics and Gynecology, St. Reterburgsky State Pediatric

Medical University marinavladi73@mail.ru

Sergey Nikolaevich Gaidukov MD, Professor, Head of Department of Obstetrics and Gynecology, St. Reterburgsky

State Pediatric Medical University gaiducovsn@rambler.ru

\title{
PATHOGENETIC SUBSTANTIATION TESTS FOR IMPAIRMENT OF TUBAL PATENCY FOR DETERMINING THE INDICATIONS FOR OPERATIVE TREATMENT TUBOPERITONEAL INFERTILITY
}

\begin{abstract}
The relevance of tubal-peritoneal infertility problems due to its impact not only on women's reproductive health, but has sodialnoe, demographic and economic importance. The main area of research is to increase the effectiveness of surgical treatment of tubal-peritoneal infertility using microsurgical endovileohirurgicheskih and innovative technologies. Of great importance is a reliable diagnosis of interruption of tubal patency as a criterion tuboperitoneal infertility. Hysterosalpingography, gidrogisterosalpingoehografiya, contrast ehogisterosalpingografiya, multislice computed gisterosalpingografyui are the main non-invasive methods of diagnosis interruption of tubal patency, aimed at identifying organic changes in the fallopian tubes new direction in the diagnosis of tubal infertility is the study of the function of the reflex interruption of tubal patency. A method for the diagnosis of tubal patency in infertility atent RF 2522395), comprising carrying out a dynamic contrast ehogisterosalpingografii blockade on the background of pathological reflexes, leading to disruption of cross-reflex isthmic parts of the fallopian tubes. The theoretical basis is the principle of convergence and divergence pathological impulses, against which develops functional disorders in organs not involved in the primary pathological process. In a study of 89 women, aimed at operative treatment for tubal peritoneal infertility by the proposed method in $23(25,4 \pm 4,5 \%)$ patients had contrast flow into the fallopian tubes and then into the abdominal cavity. At the same time $7(7,9 \pm 2,7 \%)$ noted patency restoration of one of the fallopian tubes. That is, the use of contrast against the background of the blockade ehogisterosalpingografii pathological impulses to isthmic region of the fallopian tubes department revealed functional reflex cause violation of their patency. A promising area is the development of methods to assess the transport function of the fallopian tubes.

Key words: tubo-peritoneal infertility, tubal patency, ehogisterosalpingografiya, the transport function of the fallopian tubes, reflektornle tubal infertility, surgical treatment.

Language: Russian

Citation: Salekhov SA, Nurmukhambetova BR, Konovalova MV, Prohorovich TI, Gaidukov SN (2016) PATHOGENETIC SUBSTANTIATION TESTS FOR IMPAIRMENT OF TUBAL PATENCY FOR
\end{abstract}

ISPC Generalization of scientific results, 
DETERMINING THE INDICATIONS FOR OPERATIVE TREATMENT TUBOPERITONEAL INFERTILITY.

ISJ Theoretical \& Applied Science, 12 (44): 29-33.
Soi: http://s-o-i.org/1.1/TAS-12-44-6
Doi: crossef http://dx.doi.org/10.15863/TAS.2016.12.44.6

\section{ПАТОГЕНЕТИЧЕСКОЕ ОБОСНОВАНИЕ ИССЛЕДОВАНИЯ ФУНКЦИОНАЛЬНОГО НАРУШЕНИЯ ПРОХОДИМОСТИ МАТОЧНЫХ ТРУБ ПРИ ОПРЕДЕЛЕНИИ ПОКАЗАНИЙ К ОПЕРАТИВНОМУ ЛЕЧЕНИЮ ТРУБНО-ПЕРИТОНЕАЛЬНОГО БЕСПЛОДИЯ}

Аннотация: Актуальность проблемы трубно-перитонеального бесплодия обусловлена с его влиянием не только на репродуктивное здоровье женщины, но имеет содиальное, демографическое и экономическое значение. Основным направлением исследований является повышение эффективности хирургического лечения трубно-перитонеального бесплодия с использованием микрохирургических $и$ эндовилеохирургических инноваччинных технологий. Большое значение имеет достоверная диагностика нарушения проходимости маточных труб как критерий трубно-перитонеального бесплодия. Гистеросальпингография, гидрогистеросальпингоэхография, контрастная эхогистеросальпингография, мультиспиральную компьютерную гистеросальпингографюи являются основными неинвазивными методами диагностики нарушения проходимости маточных труб, направленных на выявление органических изменений в маточных трубах Новым направлением в диагностике трубного бесплодия является исследование функиионального рефлекторного нарушения проходимости маточных труб. Разработан способ диагностики проходимости маточных труб при бесплодии атент РФ 2522395), включающий проведение динамической контрастной эхогистеросальпингографии на фоне блокады патологических рефлексов, приводящих к рефлекторному нарушению проходимости истмических отделов маточных труб. При обследовании 89 женщин, направленных на оперативное лечение по поводу трубноперитонеального бесплодия по предложенной методике у 23 (25,4士4,5\%) пациенток отмечалось поступление контраста в маточные трубы, а затем и в брюшную полость. При этом у 7 (7,9士2,7\%) отмечалось восстановление проходимости одной из маточных труб. То есть, применение контрастной эхогистеросальпингографии на фоне блокады патологической импульсации к зоне истмического отдела маточных труб позволило выявить функцииональную рефлекторную причину нарушения их проходимости. Перспективным направлением является разработка методов оценки транспортной функции маточных труб.

Ключевые слова: трубно-перитонеальное бесплодие, нарушение проходимости маточных труб, эхогистеросальпингография, транспортная функция маточных труб, рефлекторнле трубное бесплодие, хирургическое лечение.

\section{Введение}

Значимость нарушения репродуктивной функции женщины настолько велика, поскольку бесплодие влияет на медицинские, социальные, экономические, психологические аспекты современного общества, вне зависимости от того состоит ли женщина в браке или нет, что переоценить ее практически невозможно.

Значимость нарушения репродуктивной функции женщины настолько велика, поскольку бесплодие влияет на медицинские, социальные, экономические, психологические аспекты современного общества, вне зависимости от того состоит ли женщина в браке или нет, что переоценить ее практически невозможно.

Именно поэтому исследования, направленные на сохранение и восстановление репродуктивного здоровья женщины были и остаются приоритетными не только в гинекологии, но и медицины в целом $[1,2,3]$.

Причинами трубного бесплодия могут быть нарушение трофики маточных труб вследствие морфологических изменений после воспалительных процессов, что может привести к нарушению их проходимости и транспортной функции $[4 ; 5 ; 6]$.

Большое внимание в решении проблемы трубно-перитонеального бесплодия принадлежит вспомогательным репродуктивным технологиям и хирургическому лечению [2, 7; 8]. Среди хирургического лечения для лечения данной патологии широкое распространение нашли микрохирургические [2, 9] и лапароскопические операции $[10,11]$, цель которых обеспечить восстановление проходимости маточных труб. При этом именно достоверные результаты предварительного обследования, направленные на выявление нарушения проходимости маточных труб позволяют определить показания к хирургическому лечению трубноперитонеального бесплодия.

На смену традиционному рентгенологическому исследованию гистеросальпингографии (ГСГ) пришли технологии, основанные на проведении ультразвукового исследования и оценке проходимости маточных труб в режиме реального времени. 
В настоящее время, для диагностики нарушения проходимости маточных труб применяют гидрогистеросальпингоэхографию (ГГСЭГ) [12, 13; 14] или мультиспиральную компьютерную гистеросальпингографюи (МСКТГСГ) [15]. При этом проведения ГСГ и ГГСЭГ на фоне спазмолитиков создает предпосылки для снятия мышечного спазма и увеличения достоверности результатов исследования. [12].

Заслуживает внимания то, что эти методы направлены на выявление органических нарушений проходимости маточных труб, но не позволяют оценить значимость функциональных факторов в получении ложно положительных результатов. Более того, применение спазмолитиков перед исследованием не оказывает влияния на развитие функциональных нарушений, обусловленных реализацией периферических патологических (генитальногенитальный, висцеро-генитальный, париетогенитальный, спинально-генитальный) тормозных рефлексов.

Соответственно, применение спазмолитиков перед исследованием проходимости маточных труб не оказывает существенного влияния на расширение диагностических возможностей при оценке показаний к оперативному лечению.

В то же время, само применение спазмолитиков перед исследованием проходимости маточных труб свидетельствует о том, что изначально предполагается нейтрализация влияния функционального рефлекторного фактора для получения достоверных результатов.

Цель: обоснование патогенетической целесообразности проведения мероприятий, направленных на нейтрализацию периферических патологических тормозных рефлексов при диагностике нарушения проходимости маточных труб.

\section{Материалы и методы}

Теоретическое обоснование концепции функционального рефлекторного нарушения проходимости маточных труб базировалось на развитии висцеро-висцеральных тормозных рефлексов, при которых, за счет реализации принципов конвергенции и дивергенции патологической импульсации отмечалось нарушении функционального состояния органов, ранее не вовлеченных в патологический процесс.

На основании этого были разработаны принципы дифференциальной диагностики различных вариантов кардиально-абдоминальных и кардиально-спинальных рефлексов с кардиогенной ишемией миокарда $[16 ; 17 ; 18 ; 19]$ и эффективности периферических блокад при коррекции послеоперационных нарушениях функции кишечника [20;21].

В основу клинического раздела нашего исследования положены результаты исследования проходимости маточных труб у 89 женщин репродуктивного возраста, направленных на реконструктивновосстановительное оперативное лечение по поводу трубно-перитонеального бесплодия.

Возраст женщин колебался от 23 до 35 лет и в среднем составил 29,2 23,4 года. В анамнезе они неоднократно болели неспецифическими воспалительными заболеваниями придатков матки (НВЗПС).

Соответственно, перед направлением на лапароскопические реконструктивновосстановительные операции все женщины прошли обследование на урогенитальные инфекции (УГИ) (хламидиоз, микоплазмоз, уреаплазмоз, гарднерелез, трихомонох, ВПГ, ЦМВ, кандидоз и др). При необходимости им проводилось лечение УГИ и, связанных с ними НВЗПМ.

Таким образом, при поступлении у женщин направленных на оперативное лечение отсутствовали неспецифические воспалительные гинекологические заболевания в стадии обострения.

Показаниями для направления на реконструктивныеоперации по поводу трубноперитонеального бесплодия являлись результаты эхогистеросальпингографии (ЭГСГ) на фоне предварительного введения спазмалитиков (ношпа, папаверин), при проведении которой было выявлено нарушение проходимости маточных труб.

Bce женщины, рассматриваемые в этом сообщении дали письменное информированное согласие на дополнительное обследование, включающее исследование крови и материала из влагалища, шейки матки и уретры на урогенитальную инфекцию и исследования проходимости маточных труб по разработанной нами методике (Способ диагностики проходимости маточных труб при бесплодии; патент РФ 2522395, опубликовано: 10.07.2014).

При обследовании на урогенитальные инфекции у всех больных, рассматриваемых в нашей работе при исследовании материала из половых путей методом ПЦР антиген возбудителей УГИ (хламидиоз, микоплазмоз, уреаплазмоз, гарднерелез, трихомонох, ВПГ, ЦМВ, кандидоз) отсутствовал, а в крови антитела $\operatorname{Ig}$ М и $\operatorname{IgA}$ к этим возбудителям так же не были обнаружены (ИФА).

Способ диагностики проходимости маточных труб при бесплодии заключается в том, что проводится контрастная ЭГСГ на фоне введения в полость матки анэхогенного или 
гиперэхогенного контраста. При этом во время проведения исследования при выявлении нарушения проходимости маточных труб производится двухсторонняя блокада круглой связки матки $10,0-15,0$ мл $0,25 \%$ раствора новокаина с каждой стороны либо другим раствором, обладающим местно-анестезирующим действием. После этого, иногда практически во время блокады, либо в течение 5-10 минут после нее при наличии рефлекторного нарушения проходимости маточных труб на фоне спазма миометрия в зоне их истмических отделов отмечается контрастирование маточных труб и поступление контраста в брюшную полость.

Таким образом, разработанный нами способ диагностики проходимости маточных труб при бесплодии за счет ликвидации спазма в углах матки обеспечивает восстановление проходимости маточных труб, что позволяет повысить качество диагностики проходимости маточных труб при бесплодии и, в ряде случаев отказаться от проведения реконструктивновосстановительных операций на них.

\section{Результаты исследования}

Результаты предварительного обследования 89 женщин, направленных на реконструктивновосстановительные операции по поводу трубноперитонеального бесплодия, у которых при проведении ЭГСГ на фоне предварительного введения спазмалитиков расценивались как контрольные результаты при оценке результатов обследования, полученных при проведении контрастной ЭГСГ по разработанной нами методике.

Во время контрастной ЭГСГ до проведения двухсторонней блокады круглых связок матки во всех случаях поступления контраста в маточные трубы и брюшную полость не отмечалось, что совпадало с предварительными результатами при направлении на оперативное лечение.

В отличие от этого, после проведения двухсторонней блокады круглых связок матки у $23(25,4 \pm 4,5 \%)$ пациенток отмечалось поступление контраста в маточные трубы, а затем

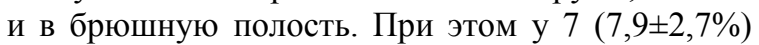
отмечалось восстановление проходимости одной

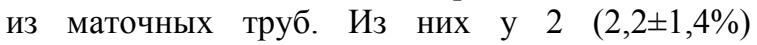
восстановилась проходимость правой, а у 5 $(5,6 \pm 2,3 \%)$ левой маточной трубы.

У остальных $16(17,5 \pm 3,9 \%)$ восстановление проходимость отмечалось с двух сторон.

Таким образом, у $23(25,4 \pm 4,5 \%)$ пациенток результаты предварительного обследования ЭГСГ были уточнены, что позволило провести коррекцию дальнейшей тактики лечения этих пациентов.

В то же время, восстановления проходимости маточных труб не является критерием функционального состояния маточных труб в полном объеме. При этом необходимо провести анализ транспортной функции маточных труб.

Так, выявление нарушения транспортной функции маточных труб при сохранении их проходимости будет свидетельствовать о необходимости проведения мероприятий направленных на ее восстановление и/или постановке вопроса о направлении на экстракорпоральное оплодотворение (ЭКО), что является перспективным направлением в изучении функционального трубного бесплодия.

\section{References:}

1. Kulakov VI (2007) Novyie tehnologii i nauchnyie prioritetyi $\mathrm{v}$ akusherstve $\mathrm{i}$ ginekologii // Ginekologiya. 2007. T. 9. \#1. p. 5-7.

2. Yakovleva NV (2014) Hirurgicheskoe lechenie trubnogo besplodiya: problemyi i resheniya / N.V. Yakovleva // Vestnik novyih meditsinskih tehnologiy - 2014. - \# 1 / tom XX / 2014. p. 121-127.

3. Tkachenko LV (2009) Vosstanovlenie reproduktivnogo zdorovya u zhenschin maloinvazivnyimi metodami / L. V. Tkachenko, M. Yu. Guschina, O. A. Kolesnichenko // Vestnik Volgogradskogo gosudarstvennogo meditsinskogo universiteta. - 2009. - \# 3 (31) / 2009 - p. $92-96$

4. Salehov SA (2013) Diagnostika funktsionalnoy neprohodimosti matochnyih trub / S.A. Salehov, S.N. Gaydukov, E.V. Gatin R.F. Ischenko, T.A. Alekseeva // Aktualnyie voprosyi akusherstva, ginekologii i perinatalogii. - 2013. - T. 55. - \# 1-3. - p. 391-392.

5. Shaw JL (2010) Current knowledge of the aetiology of human tubal ectopic pregnancy / J.L. Shaw, S.K. Dey, H.O. Critchley et al. // Hum Reprod Update. - 2010. - Vol. 16. - p. 432-444. 


\begin{tabular}{|c|c|c|c|c|c|c|}
\hline Impact Factor: & $\begin{array}{l}\text { ISRA (India) } \\
\text { ISI (Dubai, UAE } \\
\text { GIF (Australia) } \\
\text { JIF }\end{array}$ & $\begin{array}{l}=1.344 \\
=0.829 \\
=0.564 \\
=1.500\end{array}$ & $\begin{array}{l}\text { SIS (USA) } \\
\text { PИНЦ (Russia) } \\
\text { ESJI (KZ) } \\
\text { SJIF (Morocco) }\end{array}$ & $\begin{array}{l}=0.912 \\
=0.234 \\
=1.042 \\
=2.031\end{array}$ & $\begin{array}{l}\text { ICV (Poland) } \\
\text { PIF (India) } \\
\text { IBI (India) }\end{array}$ & $\begin{array}{l}=6.630 \\
=1.940 \\
=4.260\end{array}$ \\
\hline
\end{tabular}

6. Salehov SA, Gaydukov SN, Amitahunova LA, Dmitrieva OY (2009) Sostoyanie vaskulyarizatsii matochnyih trub pri vospalitelnyih zabolevaniyah pridatkov matki i trubnoy beremennosti / S.A. Salehov, S.N. Gaydukov, L.A. Amitahunova. O.Yu. Dmitrieva // Vestnik Kyirgyizsko-Rossiyskogo Slavyanskogo universiteta. 2009. \# 7. p.118122.

7. El Sharafi (2015) Vosstanovlenie reproduktivnoy funktsii u zhenschin, perenesshih rekonstruktivno-plasticheskie operatsii na matochnyih trubah / El Sharafi, O. L. Belskih, M. D. Mihaylova, O. M. Vorotneva II Fundamentalnyie issledovaniya - 2015. - \# 11. - p. 194-198.

8. Kulkarni G (2014) Survey of reasons for discontinuation from in vitro fertilization treatment among couples attending infertility clinic / G Kulkarni, NC Mohanty, IR Mohanty et al. // J Hum Reprod Sci. 2014 Oct-Dec. no. 7(4). p. 249-54 PMID: 25624660.

9. Schippert C (2010) Reconstructive, organpreserving microsurgery in tubal infertility: still alternative to in vitro fertilization / C. Schippert, C. Bassler, P. Soergel et al. // Fertil. Steril. - 2010. - Vol. 93. - N 4. - p. 13591361.

10. Yakovleva NV (2013) Znachenie endoskopicheskih tehnologiy $\mathrm{v}$ diagnostike $\mathrm{i}$ vyibore metoda lecheniya patsientok s trubnoperitonealnyim besplodiem / N.V. Yakovleva // Mat i ditya v Kuzbasse 2013. - \# 2. - p. 31-37.

11. Novy MJ (1995) Tubal surgery of IVF - making the best choice in the 1990s. Int. J. Fertil. Menopausal Stud. 1995; 40(6): 292-297.

12. Kuzmenko ET (2005) Rezultatyi primeneniya neinvazivnyih metodov diagnostiki trubnogo faktora besplodiya / E. T. Kuzmenko, A. V.Labyigina // Byulleten Vostochno-Sibirskogo nauchnogo tsentra Sibirskogo otdeleniya Rossiyskoy akademii meditsinskih nauk - 2005. - \# 5 (43) 2005. - p. 53-55.

13. Nazarenko TA (2004) Sovremennyie podhodyi $\mathrm{k}$ diagnostike $\mathrm{i}$ lecheniyu besplodiya $\mathrm{u}$ zhenschin / T.A. Nazarenko, E.R. Duryanyan, S.G. Perminova // Zhurnal dlya prakticheskih vrachey. Ginekologiya. - 2004. - T. 6, \# 6. - p. 323- 325.

14. Astafeva OV (2010) Ehogisterosalpingografiya kak metod otsenki anatomicheskogo i funktsionalnogo sostoyaniya matochnyih trub / O. V. Astafeva, A.V. Pomortsev, A.V.
Tsyibulnikov i soavt. // Kubanskiy nauchnyiy meditsinskiy vestnik - 2010. - \# 2. - p. 10-15.

15. Horoshun ND (2012) Vozmozhnosti multispiralnoy kompyuternoy gisterosalpingografii $\mathrm{V}$ diagnostike trubnoperitonealnogo besplodiya / N.D. Horoshun, L. V. Adamyan, K.D. Murvatov i soavt. // Vestnik Volgogradskogo gosudarstvennogo meditsinskogo universiteta . - 2012. - \# 3 (43). - p. 81-84.

16. (2002) Sposob differentsialnoy diagnostiki kardiogennoy ishemii miokarda i ostrogo holetsistita. / A.I. Korabelnikov, S.A. Salehov, M.P. Salehova, V.R. Veber // Patent RF \# 2190347; opublikovan 10.10.2002.

17. (2014) Sposob differentsialnoy diagnostiki i anorektalno-kardialnogotormoznogo refleksa / Salehov S.A., Korabelnikov A.I., Veber V.R., Salehova M.P., Adilbekov E.A. // Patent RF \# 2535623 opublikovan 20.12.2014

18. (2014) Sposob differentsialnoy diagnostiki kardiogennoy ishemii miokarda i enteralnokardialnogo tormoznogo refleksa / V.R. Veber, M.P. Salehova, A.I. Korabelnikov, S.A. Salehov i soavt.// Patent RF \# 2535621; opublikovan 20.12.2014.

19. (2015) Sposob differentsialnoy diagnostiki kardiogennoy ishemii miokarda i ileotsekalnokardialnogo tormoznogo refleksa / A.I. Korabelnikov, V.R. Veber, M.P. Salehova, S.A. Salehov i soavt.// Patent RF \# 2541826; opublikovan 20.02.2015.

20. (2015) Sposob differentsialnoy diagnostiki kardiogennoy ishemii miokarda i genitalnokardialnogo tormoznogo refleksa / M.P. Salehova, A.I. Korabelnikov, V.R. Veber, S.A. Salehov i soavt.// Patent RF \# 2550000; opublikovan 10.05.2015.

21. (2016) Sposob differentsialnoy diagnostiki kardiogennoy ishemii miokarda i spinalnokardialnogo tormoznogo refleksa / S.A. Salehov, V.R. Veber, A.I. Korabelnikov i soavt.// Patent RF \# 2602952; opublikovan 20.11.2016.

22. (2016) Sposob kateterizatsii i blokadyi zabryushinnyih nervnyih obrazovaniy / S.A. Salehov, V.R. Veber, A.I. Korabelnikov i soavt.// Patent RF \# 2602173; opublikovan 10.11.2016.

23. (2014) Sposob diagnostiki prohodimosti matochnyih trub pri besplodii / S.A. Salehov, S.N. Gaydukov, E.V. Ischenko i soavt. // Patent RF \# 2522395:; opublikovan 10.07.2014. 